\title{
Uncoupling resistance to cancer immunotherapy
}

cs

treatment
of tumour-
engrafted
mice with a
combination of
rosiglitazone
and anti-PD1
antibodies
significantly
prolonged
survival

versus either

treatment

alone

D)

Immune checkpoint inhibitors unleash the power of a patient's immune system on tumour cells and have dramatically improved outcomes in some cancer types. However, the effectiveness of this therapy is limited in many cases by poor immune cell infiltration and a non-inflammatory tumour microenvironment. A new study in Nature Immunology suggests that pharmacological induction of mitochondrial uncoupling protein 2 (UCP2) expression in tumour cells can promote immune cell infiltration and improve responses to checkpoint inhibitor therapy, highlighting a strategy to turn up the heat on immunologically cold tumours.

Cheng et al. began by analysing gene expression patterns of patients with melanoma in The Cancer Genome Atlas (TCGA) cohort. They used these data to divide the patients into two groups according to whether expression signatures indicated a high or low $\mathrm{T}$ cell antitumour immune response.

Among the top gene hits in patients with high $\mathrm{T}$ cell antitumour responses, UCP2 emerged as the front-running metabolic enzyme. Moreover, UCP2 mRNA levels correlated with prolonged patient survival. Expression of UCP2 in melanoma cells was positively correlated with the level of CD8A mRNA in tumourinfiltrating lymphocytes, pointing to a link between UCP2 and CD8 ${ }^{+}$ $\mathrm{T}$ cell responses.

Immunohistochemical staining of melanoma sections from a validation cohort further showed that increased expression of UCP2 was associated with higher $\mathrm{T}$ cell antitumour scores.

In transcriptome analysis of TCGA patients with melanoma, the researchers found UCP2 expression to be positively correlated with genes that control interferon- $\gamma$ signalling, migration of dendritic cells (DCs) and recruitment of T cells, suggesting the promotion of an immune-stimulatory tumour microenvironment. Consistent with these findings, immunostaining showed higher frequencies of DCs in melanomas expressing UCP2.

To further probe the role of $\mathrm{UCP} 2$ in antitumour responses, Cheng et al. turned to studies in mouse models. In mice engrafted with a melanoma cell line that exhibits doxycycline-inducible expression of UCP2, induction of UCP2 considerably suppressed melanoma growth. Overexpression of UCP2 promoted tumour infiltration of $\mathrm{CD}^{+} \mathrm{T}$ cells and natural killer cells, with no effect on $\mathrm{CD}^{+} \mathrm{T}$ cells, regulatory $\mathrm{T}$ cells or B cells.

Mice engrafted with UCP2overexpressing tumours showed improved vascular integrity and increased vessel size compared with mice engrafted with control melanomas.

Antibody-mediated depletion of $\mathrm{CD}^{+} \mathrm{T}$ cells in the mice abrogated the antitumour responses induced by UCP2 overexpression, underscoring the importance of this subpopulation of T cells for UCP2-dependent effects on the tumour.
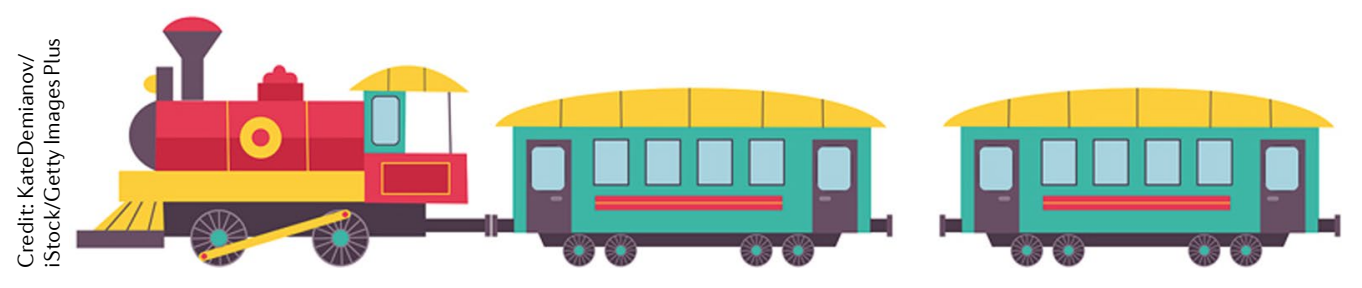

Consistent with the TCGA patient data, the investigators observed greater production of antitumour chemokines and increased DC infiltration in UCP2overexpressing tumours in mice compared with control tumours. Moreover, in transgenic mice lacking DCs, UCP2 overexpression failed to suppress tumour growth or recruit $\mathrm{CD} 8^{+} \mathrm{T}$ cells.

As UCP2 overexpression seemed to promote an inflammatory state in tumours, the authors postulated that such an environment might counteract primary resistance to checkpoint inhibitors. They therefore tested anti-PD1 antibody therapy in mice with anti-PD1resistant melanoma grafts, and indeed observed prolonged survival when UCP2 was induced in these tumours.

Peroxisome proliferator-activated receptor agonists are known to promote UCP2 expression, and include the approved diabetes drug rosiglitazone. In the current study, treatment of tumour-engrafted mice with a combination of rosiglitazone and anti-PD1 antibodies significantly prolonged survival versus either treatment alone. Conversely, in a UCP2-deficient melanoma cell line, the combination treatment failed to suppress tumour growth or prolong survival.

Together, these findings suggest a promising avenue for combination drug strategies to broaden the success of existing immunotherapies.

Katie Kingwell

ORIGINAL ARTICLE Cheng, W.-C. et al. Uncoupling protein 2 reprograms the tumor microenvironment to support the anti-tumor immune cycle. Nat. Immunol. 20, 206-217 (2019) FURTHER READING Galon, J. \& Bruni, D.

Approaches to treat immune hot, altered and cold tumours with combination immunotherapies. Nat. Rev. Drug. Discov. https://doi.org/10.1038/ s41573-018-0007-y (2019) 